\title{
NIVEL DE RIESGO POR DESBORDE E INUNDACIÓN APLICANDO UN MODELO DE FLUJO DE ESCOMBROS EN LA QUEBRADA LLUTA PARA LA MINA KARLA EN TACNA
}

\author{
RISK LEVEL FOR FLOODING AND FLOODING APPLYING A MODEL OF \\ DEBRIS FLOW MODEL IN LLUTA BASIN, FOR THE KARLA MINE, \\ TACNA
}

\author{
${ }^{1}$ Rosa Laura Quispe \\ ${ }^{1}$ Edwin Pino Vargas \\ ${ }^{2}$ Cesar Avendaño Jihuallanga
}

\section{RESUMEN}

Este trabajo busca explicar los criterios de modelación de los flujos de lodo y escombros, utilizando un modelo de análisis de flujos hiperconcentrados FLO-2D. Como datos de entrada se requiere la topografía digital del terreno, la geometría del canal, valores de la rugosidad del canal y de la planicie de inundación, hidrogramas de entrada (líquidos y sólidos), precipitación y propiedades reológicas de la mezcla agua-sedimento. La topografía para la modelación consiste de una topografía al detalle del cono de deyección y parte de la garganta. El hidrograma líquido calculado por procedimientos normales es utilizado en la modelación de flujos hiperconcentrados. Asimismo, los parámetros reológicos (viscosidad y esfuerzo de cedencia) son estimados de manera indirecta, comparando muestras de la quebrada con muestras tipo de la literatura. La quebrada modelada y simulada fue la quebrada Lluta que forma parte de la cuenca Caplina distrito Pachía, de la provincia de Tacna. Con la simulación de la quebrada, el programa FLO-2D nos reporta tirantes y velocidades del flujo de lodo, tanto en el cono de deyección como en la confluencia con el río Caplina. A partir de las salidas del programa se puede elaborar un mapa de amenaza que ayude a mitigar los efectos del huayco en la mina Karla de Tacna. El análisis de la información ha permitido establecer el aporte de sedimentos al torrente, con lo que es posible diseñar una estrategia para incrementar la seguridad aguas abajo de las quebradas en mención.

Palabras clave: Cuenca Caplina, flujos hiperconcentrados, modelo FLO-2D.

\section{ABSTRACT}

This paper attempts to explain the criteria for modeling mud and debris flows using hyperconcentrated flow analysis, the numerical model used in the FLO-2D. The digital topography of the land, the geometry of the channel, the estimated values of the roughness of the channel and the floodplain, the input hydrographs (liquids and solids), precipitation and rheological properties of the water-sediment mixture are required as input data. The topography for modeling consists of a detailed topography of the dejection cone and part of the throat. The liquid hydrograph calculated by normal procedures is used in hyperconcentrated flow modeling. In addition, the rheological parameters (viscosity and yield stress) are estimated indirectly, comparing samples from the ravine with type samples from literature. The modeled and simulated ravine was the Lluta ravine that forms part of the Caplina district of Pachía district, in the province of Tacna. With the simulation of the ravine, the FLO-2D program reports braces and mudflows velocities in both the dejection cone and in the flow with the Caplina River. From the outputs of the program, a hazard map can be prepared to help mitigate the effects of the huayco at Karla mine in Tacna.The analysis of the information has allowed to establish the content of the sediments in the torrent, with which it is possible to design a strategy to improve the safety of the waters below the streams in the mention.

Keywords: Caplina basin, hyper-concentrated flows, FLO-2D model.

${ }^{1}$ Universidad Nacional Jorge Basadre Grohmann. Tacna-Perú. E-mail: epino68@hotmail.com

${ }^{2}$ Universidad Privada de Tacna. Tacna-Perú. E-mail: neo_ceav@hotmail.com 


\section{INTRODUCCIÓN}

Una quebrada, según define Castillo (2006), es una forma topográfica de pequeña extensión producto de ciertos agentes geológicos como el viento o la fuerza de la precipitación y escorrentía en una región de terreno erosionable. Los huaycos se pueden producir en quebradas medianas (de 2,5 a $50 \mathrm{~km}^{2}$ ) y en quebradas grandes (de 50 a $150 \mathrm{~km}^{2}$ ). Según la diferencia del tamaño de la quebrada y la pendiente se pueden desarrollar diferentes tipos de flujos de escombros, como se verá más adelante. Castillo (2009) añade que los flujos de lodo y escombros son el resultado de alguna forma de colapso en el talud. Los escombros que caen como un deslizamiento colectan humedad y se mueven a lo largo de la pendiente, este se licua o se dilata conforme avanza, aumentando la movilidad del fluido.

Al respecto, Takahashi (1980) señala que los flujos de lodo y escombros son flujos antiguos conformados por una mezcla viscosa y sedimentos de todos los tamaños con acumulaciones de cantos rodados que se vuelcan en el frente de la onda y forman lóbulos, detrás de los cuales siguen los granos más finos. Según Suarez (2013), dichos flujos de lodo y escombros ocurren cuando masas de sedimento pobremente graduadas, agitadas y saturadas con agua, caen precipitadamente por efecto de la atracción de la gravedad.

Si bien existen diversas definiciones sobre flujos de escombros, básicamente se trata de una mezcla de agua con sedimento que transita hacia aguas abajo, sobre una pendiente pronunciada y que aumenta su volumen debido a rocas, arbustos y troncos que va encontrando en su camino. Los huaycos no pueden ser tratados normalmente dentro del campo de la hidráulica fluvial. Sus características marcadas grandemente por su alta concentración los hacen dignos de un estudio especial (Valderrama [2014] y Savage [1979]).

Una de las clasificaciones para flujos de escombros - basada en el tipo del mecanismo de material, el movimiento, y la concentración del sedimento- se enfoca principalmente en la banda de flujos de gravedad (mezclas de sedimento y agua) que discurren a diversas tasas de concentraciones. Costa (1987) estructura 3 grupos principales en esta clasificación: Flujos de corriente ordinario, Flujos Hiperconcentrados (Jakob y Hungr [2005]) y Flujos Granulares.

En el presente trabajo se busca realizar un análisis de riesgo y evaluar el grado de peligrosidad por desborde e inundación, al que se encuentra expuesta la mina Karla, utilizando el modelo FLO-2D y considerando, además, el comportamiento de la quebrada ante la ocurrencia de flujos de detritos bajo condiciones de descargas impuestas. En esta mina se dispone de un campamento para facilitar el desarrollo de las actividades mineras, por tanto es necesario realizar un análisis con el fin de determinar el nivel de riesgo al cual estan sujetas las personas que laboran y ocupan las instalaciones del campamento.

\section{MODELO HIDRÁULICO FLO-2D}

El movimiento de un fluido tan complejo como el que forma los aludes torrenciales no puede representarse por medio de los métodos que se aplican comúnmente para fluidos como el agua. Esto se debe a que la alta concentración de materia sólida en los aludes torrenciales hace que estos fluidos se comporten como fluidos no-newtonianos, en donde es necesario que actúe un esfuerzo superior a un esfuerzo crítico dado, para que el fluido se ponga en movimiento. Viceversa, si el esfuerzo actuante es inferior al esfuerzo crítico, el fluido se detiene (Pierson, 1986).

El modelo bidimensional de diferencias finitas FLO2D simula flujo de fluidos no-newtonianos, como aludes torrenciales, en conos de deyección. El modelo permite simular flujo en topografías complejas, tales como áreas urbanizadas y planicies de inundación, así también el intercambio de fluido entre los canales y la planicie de inundación. Puede modelarse flujo de agua, flujo hiperconcentrado de sedimentos, flujo de barro y alud torrencial. Los datos de entrada que se requiere son: la topografía digital del terreno, la geometría del canal, valores estimados de la rugosidad del canal y de la planicie de inundación, hidrogramas de entrada (líquidos y sólidos), precipitación y propiedades reológicas de la mezcla agua-sedimento (O'Brien, 2006).

\section{Ecuaciones Básicas}

Las ecuaciones que utiliza el modelo FLO-2D son las de continuidad y cantidad de movimiento, integradas en la vertical:

$$
\begin{gathered}
\frac{\boldsymbol{\partial h}}{\boldsymbol{\partial t}}+\frac{\boldsymbol{\partial h} \boldsymbol{V}_{\boldsymbol{x}}}{\boldsymbol{\partial x}}+\frac{\boldsymbol{\partial h} \boldsymbol{V}_{\boldsymbol{y}}}{\boldsymbol{\partial y}}=\boldsymbol{i} \\
S_{f x}=S_{0 x}-\frac{\partial h}{\partial x}-\frac{V_{x}}{g} \frac{\partial V_{x}}{\partial x}-\frac{V_{y}}{g} \frac{\partial V_{x}}{\partial y}-\frac{1}{g} \frac{\partial V_{x}}{\partial t} \\
S_{f x}=S_{0 y}-\frac{\partial h}{\partial y}-\frac{V_{y}}{g} \frac{\partial y}{\partial y}-\frac{V_{x}}{g} \frac{\partial V_{y}}{\partial x}-\frac{1}{g} \frac{\partial V_{y}}{\partial t}
\end{gathered}
$$

Donde: $h$ es la profundidad del flujo, $V_{x}$ y $V_{y}$ : componentes de la velocidad media, $t$ el tiempo, $g$ la aceleración de la gravedad, $S_{f}$ la pendiente de fricción, $S_{o}$ la pendiente del lecho $e_{i}$ la intensidad de la precipitación. La condición de borde aguas arriba es el hidrograma de entrada (INFLOW) y la condición de borde aguas abajo es el flujo de salida (OUTFLOW).

\section{Solución Numérica}

La forma diferencial de las ecuaciones de continuidad y de momentum en el modelo FLO-2D es resuelta mediante un esquema central de diferencias finitas. Además, el modelo emplea para el cálculo de la velocidad la ecuación de onda difusiva o la ecuación de onda dinámica.

Para la solución del método de diferencias finitas, el FLO-2D emplea un esquema explícito, el cual consiste en un esquema simple que requiere de valores pequeños de $\Delta \mathrm{x}$ y $\Delta \mathrm{t}$ para la convergencia del procedimiento numérico. Para la estabilidad numérica 
de los cálculos, una condición necesaria pero no suficiente es la condición de Courant-Friedrich-Lewy (CFL). El paso de tiempo $\Delta \mathrm{t}$ es limitado por:

$$
\Delta t=C \frac{\Delta x}{(v+c)}
$$

Donde $\mathrm{C}$ es el número de Courant $(0,3<\mathrm{C}<1,0), \Delta \mathrm{x}$ el ancho del elemento de la malla cuadrada, $v$ la velocidad promedio de la sección transversal calculada y c la celeridad de la onda calculada. Este criterio está en función de la pendiente del lecho, la descarga y el tamaño del elemento de grilla. El paso de tiempo es limitado por:

$$
\Delta t<\zeta S_{o} \Delta X^{2} / q_{o}
$$

Donde $\mathrm{q}_{\mathrm{o}}$ es la descarga unitaria, $\mathrm{S}_{\mathrm{o}}$ la pendiente del lecho, $\zeta$ es un coeficiente empírico. Los pasos de tiempo $\Delta \mathrm{t}$ generalmente varían de 0,1 segundo a 60 segundos. El modelo comienza con un paso de tiempo mínimo y lo aumenta hasta que uno de los criterios de estabilidad numérica sea excedido, entonces el paso de tiempo es disminuido. Si los criterios de estabilidad continúan siendo excedidos, entonces el paso de tiempo es disminuido hasta que el paso de tiempo mínimo sea alcanzado. Los pasos de tiempo son una función de la descarga del flujo para un elemento de grilla y de su tamaño.

\section{MODELO REOLÓGICO}

FLO-2D transita flujos de sedimentos hiperconcentrados (flujos de lodo y escombros) como un fluido continuo, prediciendo el movimiento del fluido viscoso. Para flujos de lodo (mudflows), el movimiento de la matriz fluida es gobernado por la concentración de sedimentos. Un modelo cuadrático reológico, que predice la viscosidad y el esfuerzo de cedencia como una función de la concentración de sedimentos, es empleado y los volúmenes del sedimento son monitoreados a través del sistema. Los flujos de lodo son dominados por esfuerzos viscosos y esfuerzos dispersivos, y constituyen un fenómeno muy diferente a esos procesos de carga de sedimento suspendida y carga de lecho en un transporte de sedimento convencional. El transporte de sedimento y los componentes de flujos de lodo no pueden ser simulados a la vez con el programa FLO-2D (Banda, 2014).

Takahashi \& Tsujimoto (1985) propusieron un modelo de diferencia finitas bidimensional para flujos de escombros, basado en el modelo de fluido dilatante acoplado con la resistencia del flujo de Coulomb. Inicialmente, el modelo de fluido dilatante fue derivado de la teoría de los esfuerzos dispersivos que describe los esfuerzos resultantes de la colisión de partículas de sedimento, modelo de flujos de escombros al que luego Takahashi (1980) modificó para incluir turbulencia.
O'Brien \& Julien (1988) investigaron flujos de lodo con altas concentraciones de sedimento fino en la matriz fluida. Dichos estudios mostraron que los flujos de lodo se comportan como los fluidos Bingham con bajas tasas de corte. En matrices fluidas con bajas concentraciones de sedimento, los esfuerzos turbulentos dominan en el flujo. Altas concentraciones de partículas gruesas combinadas con bajas concentraciones de partículas finas son requeridas para generar esfuerzos dispersivos. El modelo cuadrático de esfuerzo al corte describe el régimen continuo del flujo viscoso a flujo turbulento/dispersivo.

Julien \& León (2000) propusieron una formulación adimensional del modelo reológico cuadrático representado por:

$$
t^{*}=1+\left(1+T_{d}^{*}\right) a_{i} D_{v}^{*}
$$

Donde los tres parámetros adimensionales $\tau *, \mathrm{D}_{\mathrm{v}}{ }^{*} \mathrm{y} T$ * son definidos como esfuerzo cortante en exceso adimensional, tasa dipersivo-viscoso adimensional, tasa turbulento-dispersivo. Por otro lado, los esfuerzos cortantes analizados en el modelo FLO-2D pueden también escribirse de la siguiente manera:

$$
S_{f}=S_{y}+S_{v}+S_{t d}
$$

Donde $\mathrm{S}_{\mathrm{f}}$ es la pendiente de fricción total, $\mathrm{S}_{\mathrm{y}}$ la pendiente de cedencia, $S_{v}$ la pendiente viscosa y $S_{t d}$ la pendiente turbulenta- dispersiva. $\mathrm{S}_{\mathrm{v}}$ se escribe como:

$$
S_{v}=\frac{K n}{8 S \gamma_{m}} \frac{V}{h^{2}}
$$

Donde $\gamma_{\mathrm{m}}$ es el peso específico de la mezcla de sedimentos, K es el parámetro de resistencia para flujos laminares. Un valor de K de 2285 fue calibrado para el flujo de lodos de la quebrada Rudd en Utah (EE.UU.) para un área residencial y ha sido usado con buenos resultados en muchos estudios de flujos de escombros.

Tabla 1. Parámetros de resistencia del flujo laminar

\begin{tabular}{lc}
\hline \multicolumn{1}{c}{ Superficie } & Rango de K \\
\hline Concreto/asfalto & $24-108$ \\
Arena escasa & $30-120$ \\
Superficie gradual & $90-400$ \\
Arcilla escasa - suelo de marga erosionada & $100-500$ \\
Poca vegetación & $1000-4000$ \\
Hierba corta de pradera & $3000-10000$ \\
Césped de hierba añil & $7000-50000$ \\
\hline
\end{tabular}

\section{Medición de los parámetros reológicos}

O'Brien y Julien (1988) realizaron un análisis de laboratorio en muestras recolectadas de depósitos naturales de flujos de lodo en Colorado Rocky Mountain, cerca de las ciudades de Aspen y Glenwood Springs. Las propiedades de las muestras de los flujos de lodo, en términos de distribución del tamaño de 
sedimento y contenido de arcilla, son dadas en la Tabla 1. Las muestras con alto contenido de arcilla fueron ensayadas para determinar el límite líquido y el índice plástico. La matriz fluida compuesta de sedimentos finos (solo limos y arcillas) fue preparada para su ensayo en un viscosímetro; de 10 a 15 mediciones de esfuerzos cortantes versus tasa de deformación fueron realizadas para cada concentración. La concentración volumétrica fue variando añadiendo agua después de cada ensayo. Finalmente, los resultados concluyeron que la viscosidad $(\eta)$ y el esfuerzo de cedencia $(\tau y)$ se incrementan potencialmente con la concentración de sedimentos $(\mathrm{Cv})$ :

$$
\begin{aligned}
\eta & =\alpha_{1} e^{\beta_{1} C v} \\
t_{y} & =\alpha_{1} e^{\beta_{2} C v}
\end{aligned}
$$

Donde $\alpha_{1}$ y $\beta_{1}$ son coeficientes empíricos definidos por experimentos de laboratorio y $\mathrm{Cv}$ es la concentración volumétrica de sedimentos.

\begin{tabular}{|c|c|c|c|c|}
\hline \multirow{2}{*}{ Fuente } & \multicolumn{2}{|c|}{$\tau_{v}=\alpha e^{\beta C v}$} & \multicolumn{2}{|c|}{$n=\alpha e^{\beta C v}$} \\
\hline & $\alpha$ & $\boldsymbol{\beta}$ & $\alpha$ & $\beta$ \\
\hline \multicolumn{5}{|c|}{ Correlaciones de mediciones en campo } \\
\hline Aspen Pit 1 & 0,181 & 25,7 & 0,0360 & 22,1 \\
\hline Aspen Pit 2 & 2,72 & 10,4 & 0,0538 & 14,5 \\
\hline $\begin{array}{l}\text { Aspen Natu- } \\
\text { ral Soil }\end{array}$ & 0,152 & 18,7 & 0,00136 & 28,4 \\
\hline $\begin{array}{l}\text { Aspen Mine } \\
\text { Fill }\end{array}$ & 0,0473 & 21,1 & 0,128 & 12,0 \\
\hline $\begin{array}{l}\text { Aspen Wa- } \\
\text { tershed }\end{array}$ & 0,0383 & 19,6 & 0,000495 & 27,1 \\
\hline $\begin{array}{l}\text { Aspen Mine } \\
\text { Source Area }\end{array}$ & 0,291 & 14,3 & 0,000201 & 33,1 \\
\hline Glenwood 1 & 0,0345 & 20,1 & 0,00283 & 23,0 \\
\hline Glenwood 2 & 0,0765 & 16,9 & 0,0648 & 6,20 \\
\hline Glenwood 3 & 0,000707 & 29,8 & 0,00632 & 19,9 \\
\hline Glenwood 4 & 0,00172 & 29,5 & 0,000602 & 33,1 \\
\hline \multicolumn{5}{|c|}{ Correlaciones disponibles de la literatura } \\
\hline Iida (1938) & - & - & 0,0000373 & 36,6 \\
\hline $\begin{array}{l}\text { Dai et al. } \\
(1980)\end{array}$ & 2,60 & 17,48 & 0,00750 & 14,39 \\
\hline $\begin{array}{l}\text { Kang and } \\
\text { Zhang } \\
(1980)\end{array}$ & 1,75 & 7,82 & 0,0405 & 8,29 \\
\hline Qian et al. & 0,00136 & 21,2 & - & - \\
\hline (1980) & 0,050 & 15,48 & - & - \\
\hline $\begin{array}{l}\text { Chien and } \\
\mathrm{Ma}(1958)\end{array}$ & 0,0588 & $\begin{array}{l}19,1- \\
32,7\end{array}$ & - & - \\
\hline Fei (1981) & 0,166 & 25,6 & - & - \\
\hline & 0,00470 & 22,2 & - & - \\
\hline
\end{tabular}

Tabla 2. Esfuerzo de cedencia y viscosidad en función de Cv

Fuente: O'Brien y Julien (1988)

\section{CONCENTRACIÓN VOLUMÉTRICA DE SEDIMENTOS}

Para un evento de flujo de escombros, se estima una distribución de $\mathrm{Cv}$ en el tiempo partiendo de un valor cercano a 0,2 y aumentando gradualmente hasta $0,35 \mathrm{o}$ 0,45 dependiendo del tipo de sedimento de la cuenca. Si la cuenca produce flujo de lodos y escombros, entonces se debe utilizar valores cercanos a 0,45. El pico de $\mathrm{Cv}$ debe ubicarse unos minutos antes del pico del hidrograma líquido. Una clasificación dada por O'Brien en su manual de FLO-2D se observa en la Tabla 3. Eventos de avenidas grandes, como avenidas de 100 años, pueden contener demasiada agua para producir sucesos de flujos de lodo viscosos. Eventos de lluvias más pequeñas, como tormentas con periodo de retorno de 10 a 25 años, pueden tener mayor tendencia a crear flujos de lodo viscosos. La mayoría de cuencas con una historia de eventos de flujos de lodo y escombros tendrá un suministro de sedimento sustancial. Aun tormentas muy pequeñas pueden generar oleadas de flujo de lodo.

Tabla 3. Comportamiento del flujo de lodo como una

\begin{tabular}{|c|c|c|c|}
\hline \multirow{2}{*}{$\begin{array}{l}\text { Descripción } \\
\text { del Tipo de } \\
\text { Flujo }\end{array}$} & \multicolumn{2}{|c|}{$\begin{array}{l}\text { Concentración de sedi- } \\
\text { mentos }\end{array}$} & \multirow{2}{*}{$\begin{array}{l}\text { Características } \\
\text { del Flujo }\end{array}$} \\
\hline & $\begin{array}{l}\text { En Volu- } \\
\text { men }\end{array}$ & En Peso & \\
\hline \multirow{2}{*}{$\begin{array}{l}\text { Desliza- } \\
\text { mientos }\end{array}$} & $0,65-0,80$ & $0,83-0,91$ & $\begin{array}{l}\text { No hay flujo; falla } \\
\text { por deslizamiento } \\
\text { de bloques. }\end{array}$ \\
\hline & $0,55-0,65$ & $0,76-0,83$ & $\begin{array}{l}\text { Derrumbe de blo- } \\
\text { ques con defor- } \\
\text { mación interna } \\
\text { durante el desliza- } \\
\text { miento, movi- } \\
\text { miento paulatino } \\
\text { del terreno antes } \\
\text { de fallar. }\end{array}$ \\
\hline \multirow{3}{*}{$\begin{array}{l}\text { Flujos de } \\
\text { lodo } \\
\text { (Mudflow) }\end{array}$} & $0,48-0,55$ & $0,72-0,76$ & $\begin{array}{l}\text { Flujo evidente; } \\
\text { deslizamiento }\end{array}$ \\
\hline & & & $\begin{array}{l}\text { lento sostenido } \\
\text { por el flujo de } \\
\text { lodo; deformación } \\
\text { plástica bajo su } \\
\text { propio peso; } \\
\text { cohesivo; no se } \\
\text { expande en la su- } \\
\text { perficie. }\end{array}$ \\
\hline & $0,45-0,48$ & $0,69-0,72$ & $\begin{array}{l}\text { Flujo se extiende } \\
\text { sobre la superfi- } \\
\text { cie; flujo cohe- } \\
\text { sivo; algo de mez- } \\
\text { cla. }\end{array}$ \\
\hline $\begin{array}{l}\text { Avenida de } \\
\text { lodo } \\
\text { (Mud Flood) }\end{array}$ & $0,40-0,45$ & $0,65-0,69$ & $\begin{array}{l}\text { El flujo se mezcla } \\
\text { fácilmente; mues- } \\
\text { tra las propieda- } \\
\text { des fluidas en la } \\
\text { deformación; dis- } \\
\text { tribuido en la su- } \\
\text { perficie horizontal } \\
\text { pero mantiene una } \\
\text { superficie fluida } \\
\text { inclinada; partícu- } \\
\text { las grandes se de- }\end{array}$ \\
\hline
\end{tabular}
función de $C v$ 
positan (peñones); aparecen ondas pero se disipan rápidamente.

Didamente. cada de gravas y cantos rodados; se expande casi completamente sobre la superficie horizontal; aparece la superficie líquida con dos fases del fluido; las olas viajan en la superficie.

Separación del agua en la superficie; las olas viajan fácilmente; la mayoría de las arenas y gravas se han sedimentado y se mueven como arrastre de fondo Se distinguen claramente las olas y ondas; todas las partículas descansando en el fondo en condición inmóvil.

Inundación $<0,20<0,41 \quad$ Inundación de

de agua con carga

agua suspendida convencional y arrastre de fondo.

Fuente: O'Brien (2006)

La mayoría de flujos de lodo tiene un patrón distinto al desarrollo de una avenida. Inicialmente, agua limpia fluye de la cuenca lluvia-escorrentía hasta llegar al vértice del abanico. Esto puede ser seguido por una oleada u ondas frontales de lodo y escombros (40 a $50 \%$ de concentración por volumen). Cuando el pico llega, la concentración de sedimentos promedio generalmente decrece al rango de 30 a $40 \%$ por volumen. Sobre la rama decreciente del hidrograma, las oleadas de altas concentraciones pueden ocurrir.

Cuando transitamos avenidas de lodo o flujos de lodo sobre un abanico aluvial o planicies de inundación, el modelo FLO-2D conserva la continuidad para el agua y el sedimento. Para cada elemento de grilla y paso de tiempo, el cambio en los volúmenes de agua y sedimento y el cambio correspondiente en la concentración de sedimentos son calculados. Al final de la simulación, el modelo reporta la cantidad de agua y sedimento fuera del área de estudio (flujo de salida) y la cantidad de agua y sedimento remanente sobre el abanico y en el canal (flujo almacenado).

\section{EVALUACIÓN DE RIESGOS}

El análisis de riesgo o peligro de inundación en una localización específica, que incluye la afectación de las obras estructurales, es una función de la intensidad y de la probabilidad del evento (Noya, 2002). La intensidad de la crecida está definida por la profundidad y la velocidad de flujo y su probabilidad de ocurrencia se relaciona inversamente a la magnitud del evento (Banda, 2014). El riesgo de la crecida se define entonces, como una función combinada discreta de la intensidad del evento (severidad del acontecimiento) y de su período de retorno (frecuencia) y en general se acepta la categorización presentada en la tabla 4.

Tabla 4. Definición de riesgo de inundación

\begin{tabular}{cl}
\hline $\begin{array}{c}\text { NIVEL DE } \\
\text { RIESGO }\end{array}$ & \multicolumn{1}{c}{ DESCRIPCIÓN } \\
Alto & $\begin{array}{l}\text { Peligro para las personas tanto en el interior } \\
\text { como en el exterior de sus viviendas. Las es- } \\
\text { tructuras están en peligro de ser destruidas. } \\
\end{array}$ \\
& $\begin{array}{l}\text { Las personas están en peligro fuera de sus ca- } \\
\text { sas. Los edificios pueden sufrir daños y es po- } \\
\text { sible la destrucción de estructuras, depen- } \\
\text { diendo de las características de la construcción. }\end{array}$ \\
& $\begin{array}{l}\text { El peligro para las personas es bajo o no existe. } \\
\text { Los edificios pueden sufrir pequeños daños, } \\
\text { pero la inundación o la sedimentación podrían } \\
\text { afectar interiores de las estructuras. }\end{array}$ \\
\hline
\end{tabular}

Si bien las condiciones específicas en una región pueden requerir cambiar los umbrales de los niveles de peligro, frecuentemente se acepta la definición de intensidades de las crecidas de agua y de barro que se muestran en las tablas 5 y 6 . Los flujos de barros y detritos son aún más destructivos que las crecidas de agua, por lo que el criterio de intensidad para estos flujos es más conservativo(O'Brien, 2006).

Tabla 5. Definición de intensidad de crecidas de aguas, Flo-2D Users Manual (2009)

\begin{tabular}{|c|c|c|}
\hline $\begin{array}{l}\text { INTEN- } \\
\text { SIDAD } \\
\text { (m) }\end{array}$ & $\begin{array}{l}M A ́ X I M A \\
\text { PROFUNDI- } \\
D A D h(m)\end{array}$ & $\begin{array}{l}\text { PRODUCTO DE MÁ- } \\
\text { XIMA PROFUNDI- } \\
\text { DAD POR VELOCI- } \\
\text { DAD MÁXIMA }\left(\mathrm{m}^{2} / \mathrm{s}\right)\end{array}$ \\
\hline Alto & $\mathrm{h}>1,5$ & $\mathrm{vh}>1,5$ \\
\hline Media & $0,5<\mathrm{h}<1,5$ & $0,5<\mathrm{v} \mathrm{h}<1,5$ \\
\hline Baja & $\mathrm{h}<0,5$ & $\mathrm{v} \mathrm{h}<0,5$ \\
\hline
\end{tabular}

Tabla 6. Definición de intensidad de flujos o crecidas de barro, Flo-2D Users Manual (2009)

\begin{tabular}{|c|c|c|}
\hline $\begin{array}{l}\text { INTENSI- } \\
\operatorname{DAD}(\mathrm{m})\end{array}$ & $\begin{array}{l}\text { MÁXIMA PRO- } \\
\text { FUNDIDAD h (m) }\end{array}$ & $\begin{array}{l}\text { PRODUCTO DE MÁ- } \\
\text { XIMA PROFUNDI- } \\
\text { DAD POR VELOCI- } \\
\text { DAD MÁXIMA }\left(\mathrm{m}^{2} / \mathrm{s}\right)\end{array}$ \\
\hline Alto & $\mathrm{h}>1,0$ & $\mathrm{vh}>1,0$ \\
\hline Media & $\mathrm{h}<1,0$ & $\mathrm{vh}<1,0$ \\
\hline Baja & No definida & No definido \\
\hline
\end{tabular}




\section{MATERIALES Y METODOLOGÍA}

La mina Karla se encuentra emplazada en la quebrada Lluta y esta se ubica en la zona sur del Perú, en los distritos de Pachía y Palca, región Tacna, geográficamente el emplazamiento de la concesión minera en coordenadas UTM WGS 84 se muestra en la tabla 7. La ubicación de la zona de estudio la podemos apreciar en la figura 1 .

Tabla 7. Coordenadas de la concesión minera Karla

\begin{tabular}{ccc}
\hline \multicolumn{3}{c}{ CONCESIÓN MINERA KARLA DE TACNA } \\
\hline VÉRTICE & NORTE & ESTE \\
\hline 1 & 8032000 & 384000 \\
2 & 8029000 & 384000 \\
3 & 8029000 & 381000 \\
4 & 8030000 & 381000 \\
5 & 8030000 & 382000 \\
6 & 8031000 & 382000 \\
7 & 8031000 & 383000 \\
8 & 8032000 & 383000 \\
\hline
\end{tabular}

En la figura 1 se muestra el esquema secuencial de la metodología empleada en este trabajo para lograr los objetivos planteados, indicándose los pasos que se siguieron para el proceso de modelación y simulación.

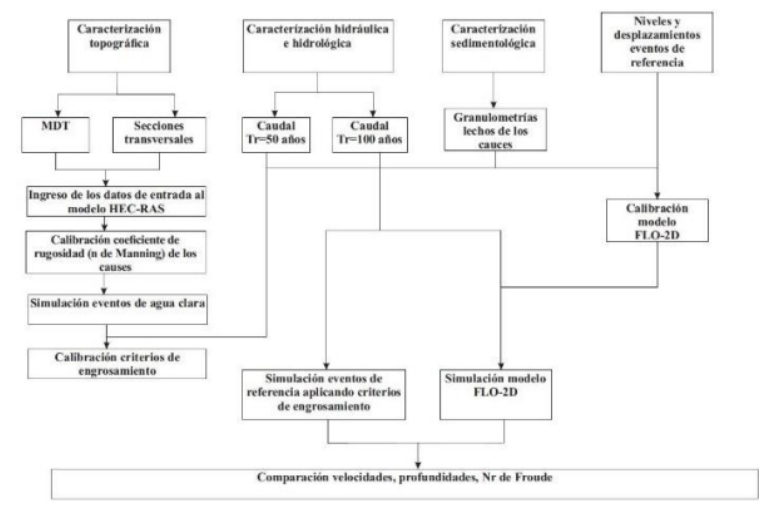

Figura 1. Esquema metodológico empleado

Se realizó la evaluación geomorfológica, geológica y la identificación de posibles marcas de antiguos flujos de escombros de la zona de estudio, verificando la información recopilada. Se excavó una calicata, luego la muestra fue llevada a laboratorio a fin de efectuar los diferentes ensayos necesarios para identificar el tipo de suelo. En la figura 2 se presenta el mapa geológico local que muestra las diferentes formaciones geológicas.

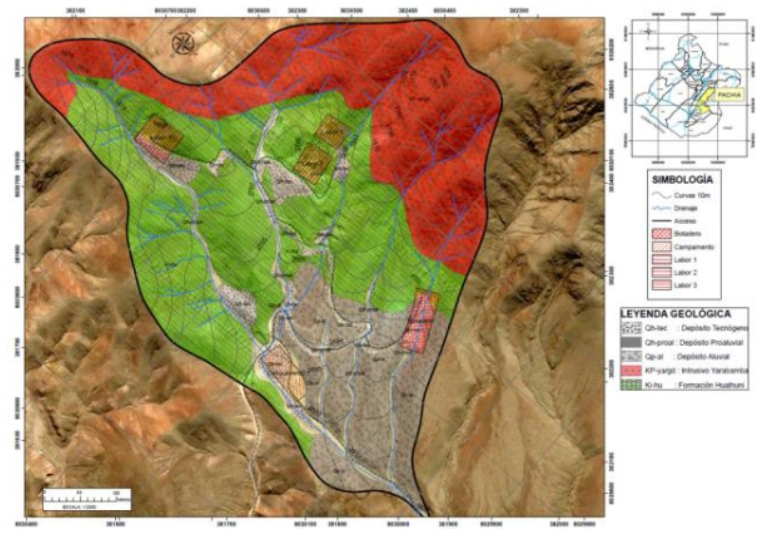

Figura 2. Mapa geológico local

La zona de estudio se encuentra ubicada dentro de la unidad morfoestructural (figura 3), denominada montañas, lomada, escarpe, colinas y depósitos de piedemonte, planicies y depresiones, y una posterior subdivisión - en base a su origen y la geometría del relieve (pendiente del terreno), carácter estructural y asociación morfogenética (fluvial, aluvial, glacial y gravitacional) - permitió establecer las unidades geomorfológicas (Valdivia et al., 2013).

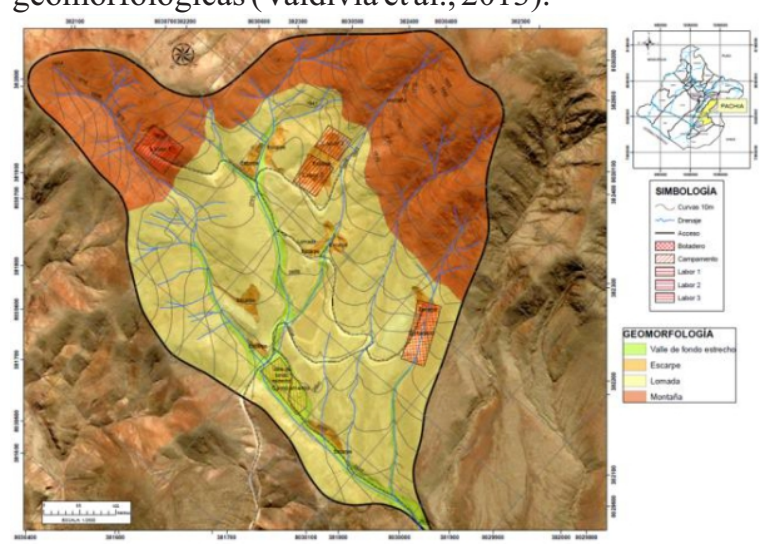

Figura 3. Mapa geomorfológico local

Asimismo se efectuó la evaluación hidrológica usando la precipitación máxima en 24 horas y se obtuvo las descargas máximas instantáneas para períodos de retorno de 50 y 100 años (figuras 4 y 5 ).

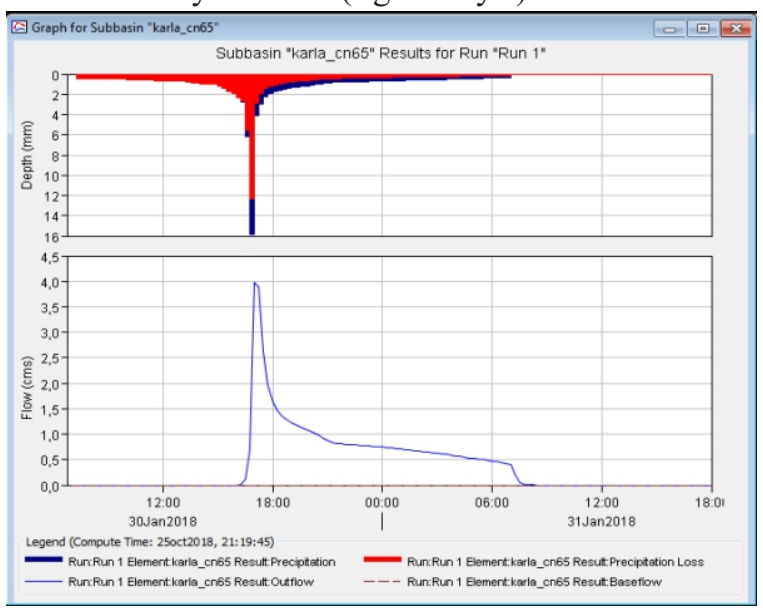

Figura 4. Hietograma e hidrograma líquido $(\mathrm{Tr}=50$ años $)$ 


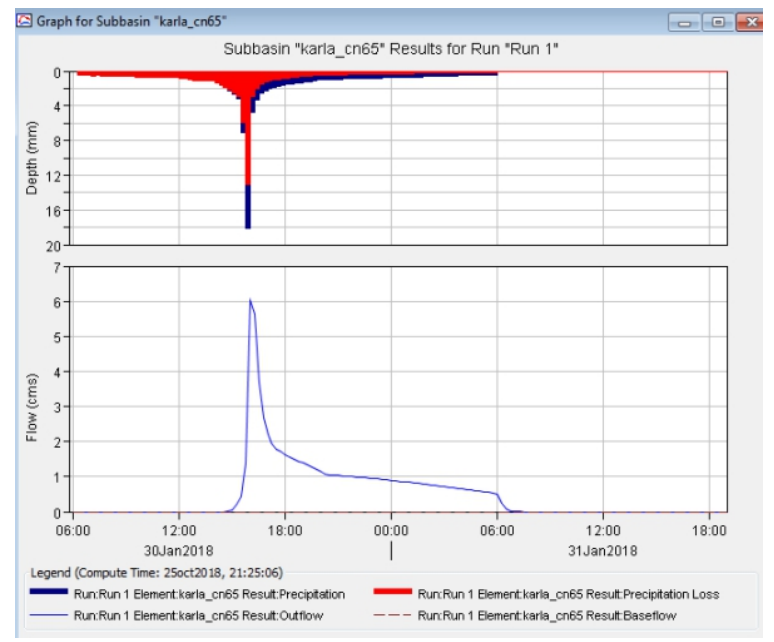

Figura 5. Hietograma e hidrograma líquido ( $\operatorname{Tr}=100$ años)

\section{SIMULACIÓN DEL FLO-2D}

Una vez conocidos los parámetros reológicos característicos de la quebrada, provenientes de la calibración del modelo, se procedió a simular bajo estas condiciones. Para ello se empleó la topografía digitalizada a escala detallada (curvas cada $10 \mathrm{~m}$ ). Para que el flujo no se estanque donde las curvas topográficas bordeen el límite de la zona de simulación, se delimitó la zona final de simulación, así el programa interpretará que no existe un impedimento para que el flujo se acumule y continúe fluyendo (figura 6).

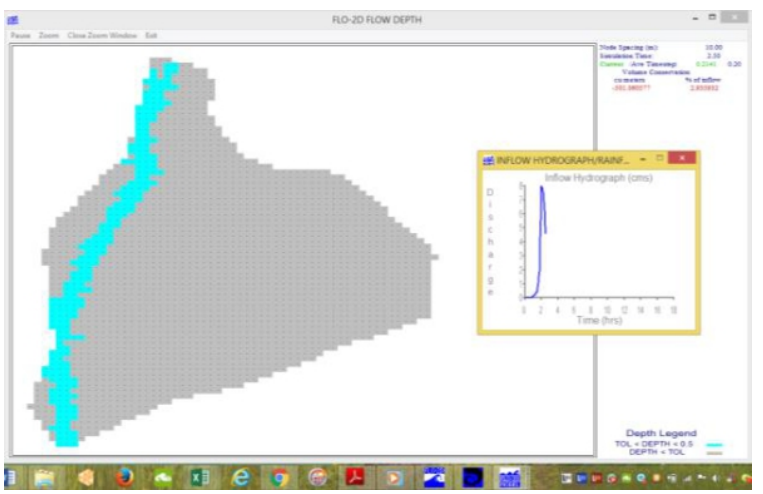

Figura 6. Salida FLO 2D, donde se aprecia en color celeste la simulación del flujo, mientras que en la ventana pequeña puede observarse cómo se desarrolla el hidrograma.

\section{RESULTADOS Y DISCUSIÓN}

\section{Escenarios Simulados}

Se ejecutaron dos simulaciones para escenarios que presenten hidrogramas líquidos con tiempos de retorno (TR) de 50 y 100 años, cuyos resultados son:

\section{Tiempo de retorno $(\mathrm{TR}=\mathbf{5 0}$ años $)$}

Este primer escenario se realizó con un hidrograma líquido, con un caudal pico de $4 \mathrm{~m}^{3} / \mathrm{s}$, para un tiempo de retorno de 50 años, y una concentración de sedimentos que varía entre $20 \%$ y $25 \%$, cuyos resultados se presentan en la tabla 8 y figuras 7 a 11 .

Tabla 8. Resultados para FLO-2D $\left(Q l p=4 \mathrm{~m}^{3} / \mathrm{s}\right)$

\begin{tabular}{lcc}
\hline \multicolumn{1}{c}{ Flujo } & Agua $\left(\mathrm{m}^{3}\right)$ & $\begin{array}{c}\text { Agua con sedi- } \\
\text { mentos }\left(\mathrm{m}^{3}\right)\end{array}$ \\
\hline $\begin{array}{l}\text { Hidrograma de } \\
\text { entrada }\end{array}$ (IN- & 46803,31 & 58844,55 \\
FLOW) & & \\
$\begin{array}{l}\text { Almacenamiento } \\
\text { dentro del área de } \\
\text { análisis }\end{array}$ & 3018,62 & 3773,43 \\
$\begin{array}{l}\text { Flujo fuera del } \\
\text { área de simula- } \\
\text { ción }\end{array}$ & 43828,84 & 55125,93 \\
\hline
\end{tabular}

El volumen total de la creciente simulada es de 0,11 $\mathrm{Hm}^{3}$, de los cuales $0,04 \mathrm{Hm}^{3}$ son agua y $0,05 \mathrm{Hm}^{3}$ corresponden a sedimento (volumen almacenado + volumen fuera del área de simulación). El área de inundación reportado por el modelo es de $23900 \mathrm{~m}^{2}$.

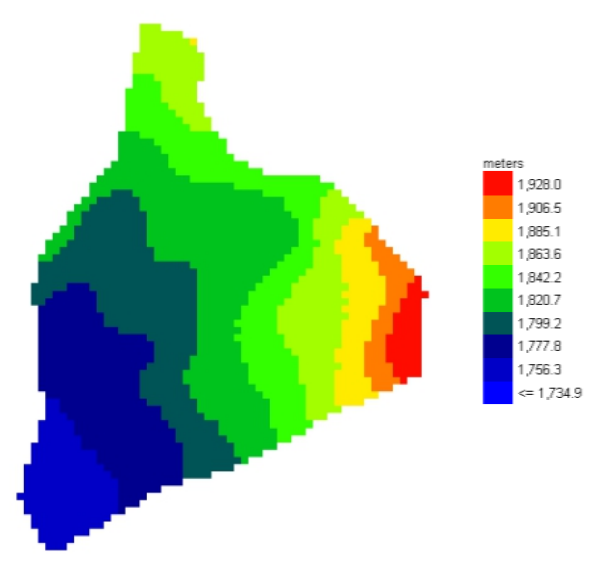

Figura 7. Elevación del terreno, TR=50 años

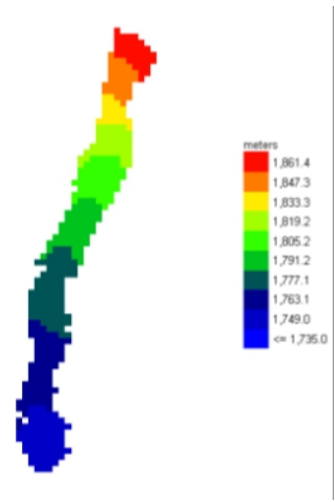

Figura 8. Máxima elevación de agua, $\mathrm{TR}=50$ años 

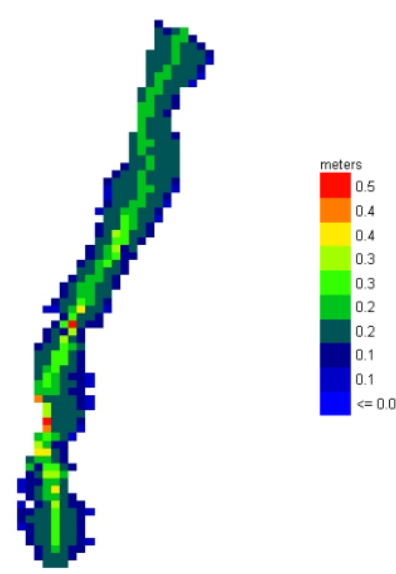

Figura 9. Profundidad máxima de flujo, $\mathrm{TR}=50$ años
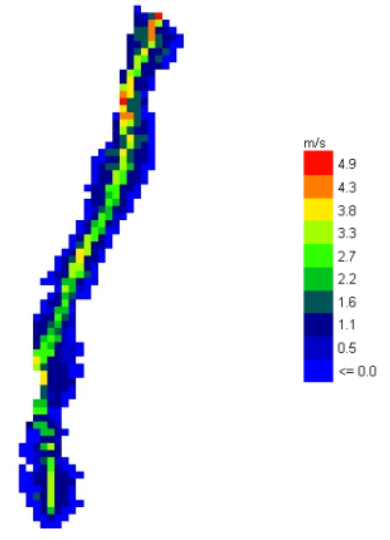

Figura 10. Velocidad máxima de flujo, $T R=50$ años

De esta forma se obtiene el mapa de peligros por flujo de detritos, con un hidrograma líquido con tiempo de retorno de 50 años y un caudal pico de $4 \mathrm{~m}^{3} / \mathrm{s}$, el cual se colorea en la grilla respectiva tomando en cuenta el comportamiento del fluido en cada elemento, como su velocidad, altura, fuerza de impacto, zonas de acumulación, entre otros.
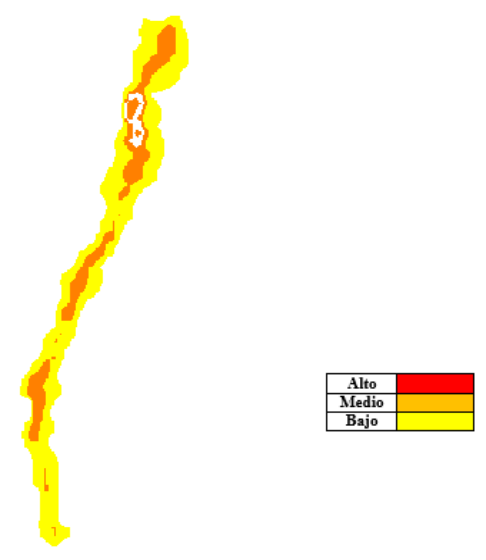

Figura 11. Mapa de peligros para $\mathrm{TR}=50$ años
Tiempo de retorno ( $T R=100$ años $)$

Este segundo escenario se realizó con un hidrograma líquido, con un caudal pico de $6 \mathrm{~m}^{3} / \mathrm{s}$, para un tiempo de retorno de 100 años, y una concentración de sedimentos que varía entre $20 \%$ y $25 \%$, cuyos resultados se presentan en la tabla 9, figura 12 .

Tabla 9. Resultados para FLO-2D $\left(Q l p=6 \mathrm{~m}^{3} / \mathrm{s}\right)$

\begin{tabular}{lccc}
\hline \multicolumn{1}{c}{ Flujo } & Agua $\left(\mathrm{m}^{3}\right)$ & $\begin{array}{c}\text { Agua con sedi- } \\
\text { mentos }\left(\mathrm{m}^{3}\right)\end{array}$ \\
\hline $\begin{array}{l}\text { Hidrograma } \\
\text { entrada }\end{array}$ & $\begin{array}{r}\text { de } \\
\text { FLOW) }\end{array}$ & 68450,99 & 86514,05 \\
& & \\
\hline $\begin{array}{l}\text { Almacenamiento } \\
\text { dentro del área de } \\
\text { análisis }\end{array}$ & 3067,42 & 3834,63 \\
$\begin{array}{l}\text { Flujo fuera del } \\
\text { área de simula- } \\
\text { ción }\end{array}$ & 65784,79 & 83180,48 \\
\hline
\end{tabular}

El volumen total de la creciente simulada es de 0,15 $\mathrm{Hm}^{3}$, de los cuales $0,06 \mathrm{Hm}^{3}$ son agua y $0,08 \mathrm{Hm}^{3}$ corresponde a sedimento (volumen almacenado + volumen fuera del área de simulación). El área de inundación reportada por el modelo es de $26800 \mathrm{~m}^{2}$.

La compilación de un mapa de riesgo de inundación es una técnica eficiente en el manejo de áreas con riesgo de inundación. Un enfoque de modelado numérico basado en escenarios se usa comúnmente para compilar un mapa de riesgo de inundación relacionado con los huaycos y para determinar los parámetros del modelo que capturan el pico de descarga (Dae-hong et al., 2015). De la misma forma se obtiene el mapa de peligros por flujo de detritos, con un hidrograma líquido con tiempo de retorno de 100 años y con un caudal pico de $6 \mathrm{~m}^{3} / \mathrm{s}$.
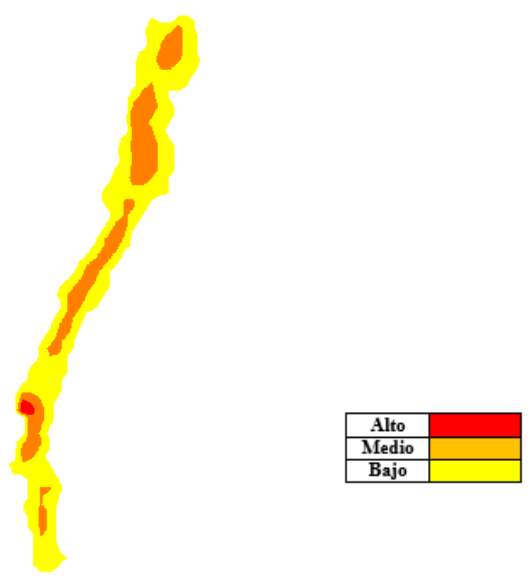

Figura 12. Mapa de peligros para $\mathrm{TR}=100$ años 


\section{CALIBRACIÓN DEL MODELOCON EVIDENCIAS DEL CAMPO}

Un parámetro de comparación se constituye por las evidencias de eventos extraordinarios vistos en campo, en los cuales se puede estimar el tirante por rasgos en las paredes de la quebrada dejada por dicho suceso (Gamión, 2014). La calibración se ha ajustado a las observaciones de campo debido a que es un parámetro posible de medir y observar in situ. A pesar de contar con una limitante como la escasa información que se dispone, es decir, solo dos tirantes, se pudo realizar la calibración respectiva.

Para calibrar el modelo con evidencias dejadas en campo se puede ejecutar dos procedimientos: El primero es realizando un análisis de comparación con el volumen de sedimentos dejado por este tipo de eventos; el segundo consiste en ajustar los tirantes del modelo con las marcas de flujo dejadas por el paso del río (Banda, 2014).

El método de calibración que utilizamos fue de la segunda forma, la cual consiste en hacer coincidir los tirantes estimados a partir de las marcas dejadas por el paso del río con los resultados del modelo, tal como ya se señaló antes. Para ello definimos puntos de control: El primero de ellos fue al tomar las evidencias que se encontraron en tramo curvo, donde se asumió que el tirante máximo dejado por el flujo alcanza una altura de los $0,31 \mathrm{~m}$ como se observa en la figura 13 .

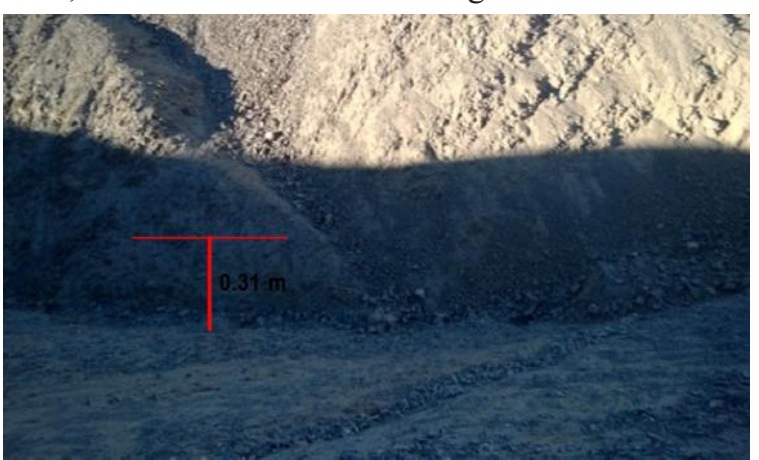

Figura 13. Marca de agua registrada en la quebrada, altura $0,31 \mathrm{~m}$

El segundo punto de control se estableció cerca al campamento de la mina Karla, donde la altura calculada es de $0,46 \mathrm{~m}$ (Figura 14).

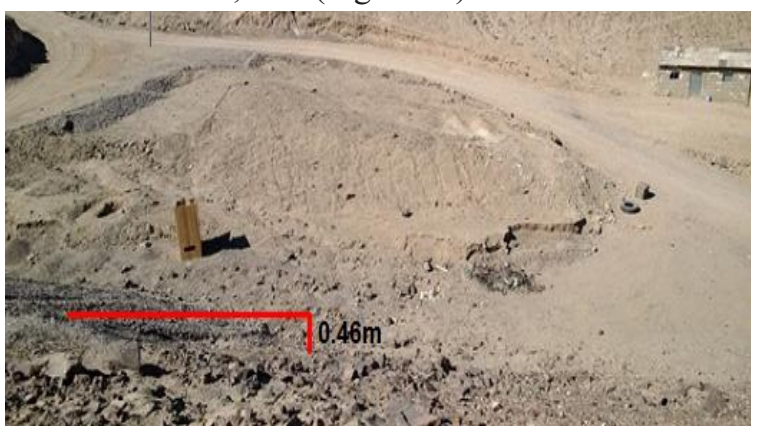

Figura 14. Marca de agua registrada en la quebrada, altura $0,46 \mathrm{~m}$
La calibración ajustada de cada altura dejada por las marcas observadas en campo, se puede ver en la Figura 14. Estos resultados son contrastados con el primer escenario, el cual presenta un mejor ajuste. Es importante tener en cuenta la sensibilidad del modelo a los parámetros físicos, pues la precisión de un modelo depende de la estructura física que describe el volumen de flujo, la formación de brechas y el progreso, las variables de entrada y los parámetros del modelo. El modelo de entrada y los parámetros son subjetivos; por lo tanto, se necesita cautela al interpretar los resultados (Lee et al., 2014). En este sentido, es importante comparar resultados con evidencias de campo (figura $15)$.

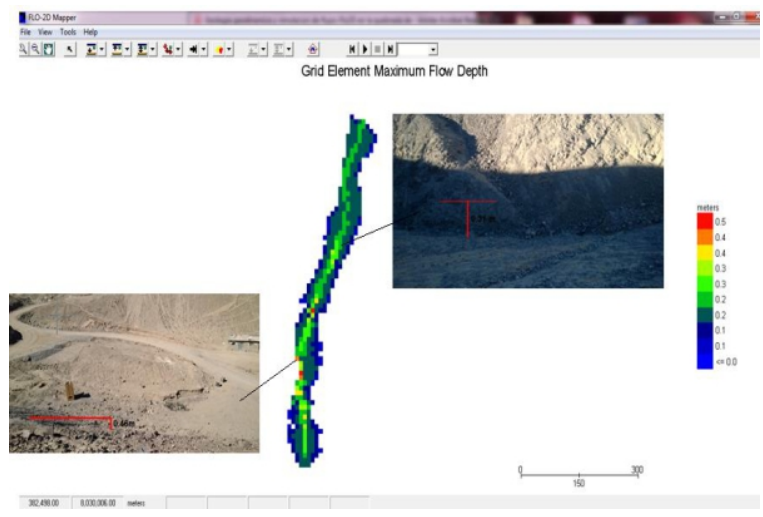

Figura 15. Comparación de resultados con evidencias de campo

Los mapas de riesgo para las simulaciones con 50 y 100 años de período de retorno se muestran en las figuras 16 y 17.

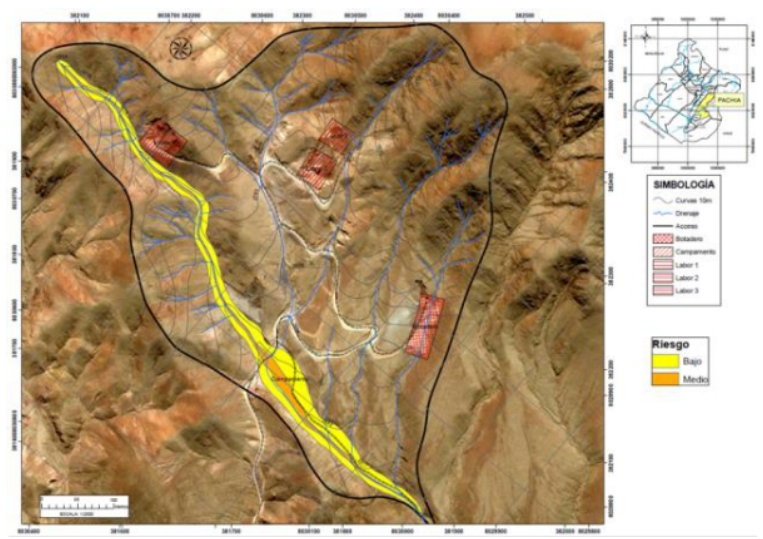

Figura 16. Mapa de peligros TR 50 años 


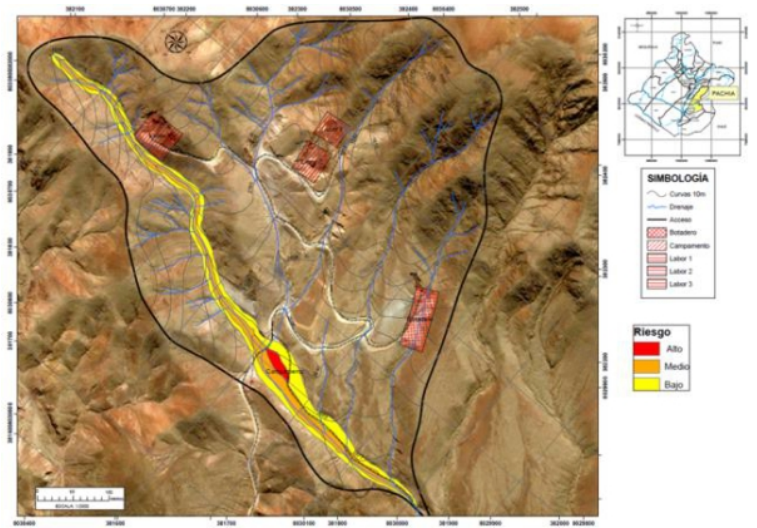

Figura 17. Mapa de peligros, TR 100 años

\section{CONCLUSIONES}

Se elaboró un mapa de peligros con el modelo FLO$2 \mathrm{D}$, en el que se plasma los resultados de las simulaciones en función de los resultados de los tirantes máximos y la velocidad del flujo. El mapa resultante permite representar la amenaza potencial que existe en cada punto de la zona, estas se diferencian entre sí mediante los colores rojo, anaranjado y amarillo según la amenaza sea alta, media o baja. Cada tipo de amenaza corresponde a una serie de daños a personas y edificaciones.

Asimismo, se realizó la zonificación por peligro de desborde o inundación para la mina Karla de Tacna; para ello se generaron dos escenarios simulados con hidrogramas líquidos con tiempos de retorno de 50 años y 100 años, con caudales de 4 y $6 \mathrm{~m}^{3} / \mathrm{s}$, respectivamente.

Para un tiempo de retorno de 50 años, los daños en estas zonas no serían considerables; dicha zonificación se ubica generalmente bordeando a las zonas de color amarillo (Peligro bajo), donde el flujo no alcanzó más que unos centímetros de altura, los daños materiales en ese caso serían mínimos o nulos.

Para un tiempo de retorno de 100 años, los daños que se producirían en estas zonas tampoco serían considerables; la zonificación en mención se ubica generalmente bordeando a las zonas de color anaranjado (Peligro medio), en las cuales el flujo no tuvo características tan complejas; puesto que la velocidad del flujo pudo haber sido alta, pero sus alturas fueron mínimas, además, la topografía y la rugosidad pudieron obstaculizar, retener o canalizar parcialmente el flujo. También se observan algunas zonas de color amarillo (Peligro bajo) donde el flujo no alcanzó más que unos centímetros de altura, los daños materiales serían mínimos o nulos, debido a que estas zonas presentarían inundaciones parciales.

Como parte de los resultados finales, se observó que para un tiempo de retorno (TR) de 50 años, la inundación tendría un volumen superior a $\operatorname{los} 11000 \mathrm{~m}^{3}$ de agua y sedimentos; en tanto que para un TR de 100 años la inundación tendría un volumen mayor a los 15 $000 \mathrm{~m}^{3}$

\section{REFERENCIAS BIBLIOGRÁFICAS}

Banda, M. (2014). Análisis comparativo de un modelo hidrodinámico unidimensional para flujos Newtonianos y no Newtonianos del río Seco, zona urbana Tacna $(70$ pp.). Tesis. Tacna: Universidad Nacional Jorge Basadre Grohmann.

Castillo, L. (2006). Aplicación de un modelo numérico de flujos de escombros y lodos en una quebrada en el Perú (193 pp.) Tesis. Lima: Universidad Nacional de Ingeniería.

Castillo, L. (2009). Aplicación del modelo numérico FLO-2D en la quebrada Cansas, Ica-Perú. Prevención de huaycos. Lima. Conferencia: Prevención de huaycos.

Costa, J. E., Wieczorek, G. F. (1987). Geological Society of America, \& Geological Society of America (Eds.). Debris flows/avalanches: process, recognition, and mitigation. Boulder, Colo: Geological Society of America.

Dae-Hong, G., Khil-Ha, Lee \& Jin-Man, Kim \& SungWook Kim. (2015). FLO-2D Simulation of the Flood Inundation Zone in the Case of Failure of the Sandae Reservoir Gyeongju, Gyeongbuk. The Journal of Engineering Geology. 25, pp. 449-458. 10.9720/kseg.2015.4.449.

Gamion, J. F. (2014). Modelo de flujo de escombros y lodos aplicando FLO 2D, caso sub cuenca del río Shullcas (176 pp.). Tesis. Huancayo: Universidad Nacional del centro del Perú.

Jakob, M. \& Hungr, O. (2005). Debris-flow hazards and related phenomena. Berlin; New York: Springer.

Julien, P. \& León, C. (2000). Mud floods, mudflows and debris flows. Classification. Rheology and structural design. Jornadas de Investigación JIFI 2000: the debris flow disaster of december 1999 in Venezuela.

Lee, K., Son, M., Ks granular materials in chutes and channels. Journal of fluid mechanics, (92 pp. Part 1).

Suarez J. (2013). Zonificación de Susceptibilidad Amenaza y Riesgo. Bucaramanga. Tomo 1: Análisis Geotécnico (527pp.).

Takahashi \& Tsujimoto. (1985). Deliniation of the debris flow haz-ardous zone by a numerical simulation method. Proc. of the intl. Symp. on erosion, Debris flow and disaster prevention, Tsukuba, Japan (pp. 547-462).

Takahashi T. (1980). "Debris flow". International Association for Hydraulic Research. AA. Balkema. Rotterdam (165pp.).

Valderrama P., Castillo L., Cardenas J., Carlotto V. \& Fidel L. (2014). Mapa de peligros y simulación del flujo FLO-2D en la quebrada Runtumayo (Cusco): alud y flujo de detritos del 12 de octubre de 2005. Lima. XIII Congreso Peruano de Geología. Sociedad Geológica del Perú.

Valdivia, J. T. (2013). Modelamiento hidrodinámico para determinar zonas vulnerables en el cauce del río seco zona urbana - Tacna (110 pp.) Tesis. Tacna: Universidad Nacional Jorge Basadre Grohmann. 